\title{
Hochschild homology and cohomology of down-up algebras
}

\author{
Sergio Chouhy, Estanislao Herscovich, and Andrea Solotar *
}

\begin{abstract}
We present a detailed computation of the cyclic and the Hochschild homology and cohomology of generic and 3-Calabi-Yau homogeneous down-up algebras. This family was defined by Benkart and Roby in [3] in their study of differential posets. Our calculations are completely explicit, by making use of the Koszul bimodule resolution and arguments similar to those appearing in [11].
\end{abstract}

2010 Mathematics Subject Classification: 16E40, 16E05, 16W50.

Keywords: Down-up algebra, homology, Hochschild, resolution.

\section{Introduction}

Motivated by the study of the algebra generated by the up and down operators in the theory of differential posets defined independently by R. Stanley in [16] and by S. Fomin in [9], or of uniform posets defined by P. Terwilliger in [18], G. Benkart and T. Roby introduced in [3] the notion of down-up algebras. They have been intensively studied in [2], [4], [6], [14], [20] among many other articles, and different kinds of generalizations have been defined [5], [7]. Since the homological invariants of an algebra provide useful tools for its description as well as for its representations, many of their homological properties were studied and in particular, a quite convenient projective resolution of the regular bimodule of a down-up algebra was constructed by S. Chouhy and A. Solotar in [8].

Let $\mathbb{K}$ be a fixed field of characteristic 0 . Given parameters $(\alpha, \beta, \gamma) \in \mathbb{K}^{3}$, the associated down-up algebra $A(\alpha, \beta, \gamma)$ is defined as the quotient of the free associative algebra $\mathbb{K}\langle u, d\rangle$ by the ideal generated by the relations

$$
\begin{aligned}
& d^{2} u-\left(\alpha d u d+\beta u d^{2}+\gamma d\right) \\
& d u^{2}-\left(\alpha u d u+\beta u^{2} d+\gamma u\right)
\end{aligned}
$$

We shall sometimes denote a particular down-up algebra $A(\alpha, \beta, \gamma)$ just by $A$ to simplify the notation.

As examples of down-up algebras, $A(2,-1,0)$ is isomorphic to the enveloping algebra of the Heisenberg-Lie algebra of dimension 3, and, for $\gamma \neq 0, A(2,-1, \gamma)$ is isomorphic to the enveloping algebra of $\mathfrak{s l}(2, \mathbb{K})$. Moreover, Benkart proved in [2] that any down-up algebra such that $(\alpha, \beta) \neq(0,0)$ is isomorphic to one of Witten's 7-parameter deformations of $\mathscr{U}(\operatorname{sl}(2, \mathbb{K}))$.

${ }^{*}$ This work has been supported by the projects UBACYT 20020130100533BA, UBACYT 20020130200169BA, PIP-CONICET 11220150100483CO, and MATHAMSUD-REPHOMOL. The second and third authors are research members of CONICET (Argentina). 
Any of these algebras has a PBW basis given by

$$
\left\{u^{i}(d u)^{j} d^{k}: i, j, k \in \mathbb{N}_{\geq 0}\right\} .
$$

Note that the down-up algebra $A(\alpha, \beta, \gamma)$ can be regarded as a $\mathbb{Z}$-graded algebra where the degrees of $u$ and $d$ are respectively 1 and -1 . We shall refer to this grading as special, and denote the special degree of an element $a \in A$ by s-deg $(a)$. In fact, $A=\bigoplus_{n \in \mathbb{Z}} A_{n}$ where $A_{n}$ is the $\mathbb{K}$-vector space spanned by the set $\left\{u^{i}(d u)^{j} d^{k} \mid i-k=n\right\}$.

It is known [6] that if $A(\alpha, \beta, \gamma)$ is isomorphic to $A\left(\alpha^{\prime}, \beta^{\prime}, \gamma^{\prime}\right)$, then

$$
\begin{aligned}
& \text { both } \alpha+\beta \text { and } \alpha^{\prime}+\beta^{\prime} \text { are } 1 \text { or different from } 1 \text {, } \\
& \text { both } \gamma \text { and } \gamma^{\prime} \text { are } 0 \text { or different from } 0 .
\end{aligned}
$$

The down-up algebra $A(\alpha, \beta, \gamma)$ is isomorphic to $A(\alpha, \beta, 1)$ for all $\gamma \neq 0$. Furthermore, if $\mathbb{K}$ is algebraically closed, P. Carvalho and I. Musson showed in [6] that $A(\alpha, \beta, \gamma)$ is isomorphic to $A\left(\alpha^{\prime}, \beta^{\prime}, \gamma^{\prime}\right)$ if and only if the following conditions hold

$$
\begin{aligned}
& \text { either } \alpha^{\prime}=\alpha \text { and } \beta^{\prime}=\beta, \text { or } \alpha^{\prime}=-\alpha^{-1} \beta \text { and } \beta^{\prime}=\beta^{-1} \text {, } \\
& \text { both } \gamma \text { and } \gamma^{\prime} \text { are } 0 \text { or different from } 0 .
\end{aligned}
$$

E. Kirkman, I. Musson and D. Passman proved in [13] that $A(\alpha, \beta, \gamma)$ is noetherian if and only it is a domain, which is tantamount to the fact that the subalgebra of $A(\alpha, \beta, \gamma)$ generated by $u d$ and $d u$ is a polynomial algebra in two indeterminates, that in turn is equivalent to $\beta \neq 0$. Under either of the previous situations, $A(\alpha, \beta, \gamma)$ is Auslander regular and its global dimension is 3 . On the other hand, it was proved by Cassidy and Shelton in [7] than, if $\mathbb{K}$ is algebraically closed, then the global dimension of $A(\alpha, \beta, \gamma)$ is always 3. Moreover, Benkart and Roby proved in [3] that the Gelfand-Kirillov dimension of a down-up algebra is 3, independently of the parameters. Since $A(\alpha, \beta, \gamma)$ is isomorphic to the opposite algebra, left and right dimensions coincide.

The centre of a down-up algebra has been computed in [14] and [20], and the first Hochschild cohomology space of a localization of some families of down-up algebras with $\gamma=0$ has been recently computed in [17], but up to now there is no description of Hochschild homology and cohomology of down-up algebras available.

The main result of this article is the computation of the complete Hochschild homology and cohomology of two families of down-up algebras with $\gamma=0$. Given $\alpha, \beta \in \mathbb{K}$, denote $r_{1}$ and $r_{2}$ the roots of the polynomial $t^{2}-\alpha t-\beta$. We define the following two cases.

(F1) Graded generic down-up algebras. The algebra $A(\alpha, \beta, 0)$ belongs to this family if and only if $(\alpha, \beta) \neq(0,0)$ and $r_{1}^{i} r_{2}^{j} \neq 1$ for all $i$ and $j$ such that $(i, j) \neq(0,0)$. We call this assumption the genericity hypothesis.

(F2) Graded 3-Calabi-Yau down-up algebras. The algebra $A(\alpha, \beta, 0)$ belongs to this family if and only if $\beta=-1$, in which case $r_{2}=r_{1}^{-1}$.

The methods we use are closely related to those used for the computation of the Hochschild and cyclic (co)homology of Yang-Mills algebras in [11], and we think that they will lead to the computation of these invariants for the other cases as well, with more involved calculations. We are not studying here the case $A(0,0,0)$ for which the resolution constructed by M. Bardzell in [1] is available.

In Section 2 we introduce some notations and basic objects such as the projective resolution of $A$ as $A$-bimodule. In case $\gamma=0$, this is the Koszul resolution. We 
state the main results of the article in Theorem 2.1 and Theorem 2.2 and leave the proofs for the subsequent sections.

In Section 3 we compute Hochschild and cyclic homology. It is clear from the resolution that $H H_{i}(A)=0$ for all $i \geq 4$. We provide explicit bases for $H H_{0}(A)$ and $\mathrm{HH}_{3}(\mathrm{~A})$ and we use a Hilbert series argument involving reduced cyclic homology and a theorem by K. Igusa in [12] to obtain the Hilbert series of $H H_{1}(A)$ and $\mathrm{HH}_{2}(A)$.

Section 4 is devoted to the Hochschild cohomology. Since $A(\alpha,-1,0)$ is 3 Calabi-Yau, we only study here algebras belonging to the family (F1). It is well known that their centre is $\mathbb{K}$ (see [14,20]). We give bases of $H H^{1}(A), H H^{2}(A)$ and $H H^{3}(A)$. This may be particularly useful for the description of the corresponding deformations.

\section{Main results}

In this section we will introduce some elements of down-up algebras with the aim of stating the main results of the article, that will be proved in the sequel.

As stated previously, we will usually denote $A(\alpha, \beta, \gamma)$ simply by $A$. We mentioned in the introduction that this algebra can be regarded as a $\mathbb{Z}$-graded algebra where the degrees of $u$ and $d$ are, 1 and -1 , respectively. We shall refer to this grading as special. If $\gamma$ is zero, the algebra $A$ has another grading that we will call usual with $u$ and $d$ both in degree 1 . We shall denote the usual degree of an element $a \in A$ by $\operatorname{deg}(a)$. Notice that the homogeneous components with respect to the usual grading are finite dimensional $\mathbb{K}$-vector spaces. For $\gamma=0, A$ is thus $\mathbb{Z}^{2}$-graded by bideg $:=($ deg, s-deg $)$.

Let $V$ be the $\mathbb{K}$-vector space spanned by the set $\{d, u\}$ and let $T(V)=\oplus_{n \geq 0} V^{\otimes n}$ be the tensor algebra of $V$ over $\mathbb{K}$. We will typically omit the tensor product symbols when writing an element of $T(V)$. Let $R$ be the subspace of $V^{\otimes 3}$ spanned by the set $\left\{d^{2} u, d u^{2}\right\}$ and let $\Omega$ be the subspace of $V^{\otimes 4}$ spanned by the element $d^{2} u^{2}$.

There is a short projective resolution of $A$ as $A$-bimodule (see [8]):

$$
0 \rightarrow A \otimes \Omega \otimes A \stackrel{\delta_{3}}{\rightarrow} A \otimes R \otimes A \stackrel{\delta_{2}}{\rightarrow} A \otimes V \otimes A \stackrel{\delta_{1}}{\rightarrow} A \otimes A \rightarrow 0
$$

where the augmentation $\delta_{0}: A \otimes A \rightarrow A$ is given by the multiplication map. The differentials are

$$
\begin{aligned}
\delta_{1}(1 \otimes v \otimes 1) & =v \otimes 1-1 \otimes v, \quad \text { for all } v \in V, \\
\delta_{2}\left(1 \otimes d^{2} u \otimes 1\right) & =1 \otimes d \otimes d u+d \otimes d \otimes u+d^{2} \otimes u \otimes 1 \\
& -\alpha(1 \otimes d \otimes u d+d \otimes u \otimes d+d u \otimes d \otimes 1) \\
& -\beta\left(1 \otimes u \otimes d^{2}+u \otimes d \otimes d+u d \otimes d \otimes 1\right) \\
& -\gamma \otimes d \otimes 1, \\
\delta_{2}\left(1 \otimes d u^{2} \otimes 1\right) & =1 \otimes d \otimes u^{2}+d \otimes u \otimes u+d u \otimes u \otimes 1 \\
& -\alpha(1 \otimes u \otimes d u+u \otimes d \otimes u+u d \otimes u \otimes 1) \\
& -\beta\left(1 \otimes u \otimes u d+u \otimes u \otimes d+u^{2} \otimes d \otimes 1\right) \\
& -\gamma \otimes u \otimes 1,
\end{aligned}
$$

and

$$
\begin{aligned}
\delta_{3}\left(1 \otimes d^{2} u^{2} \otimes 1\right) & =d \otimes d u^{2} \otimes 1+\beta \otimes d u^{2} \otimes d \\
& -1 \otimes d^{2} u \otimes u-\beta u \otimes d^{2} u \otimes 1
\end{aligned}
$$


Notice that for all $i$ the map $\delta_{i}$ is homogeneous for the special degree and the same holds for the usual degree when $\gamma=0$. Moreover, the projective resolution (2.1) is minimal in the category of graded modules if $\gamma=0$.

We will denote the complex (2.1) by $K$. It is not hard to see that if $\beta \neq 0$, then there is an isomorphism $\operatorname{Hom}_{A^{e}}\left(K, A^{e}\right) \cong A_{\sigma} \otimes_{A} K$ of complexes of $A$-bimodules, where $A^{e}=A \otimes A^{o p}$ and $\sigma$ is the automorphism determined by $\sigma(u)=-\beta^{-1} u$ and $\sigma(d)=-\beta d$. As a consequence, noetherian down-up algebras are twisted 3Calabi-Yau, and if $\beta=-1$ they are 3-Calabi-Yau. Moreover, the algebra $A(\alpha, \beta, \gamma)$ is 3-Calabi-Yau if and only if $\beta=-1$. See for example [15] and the references therein.

In the following theorem we summarize the results about Hochschild homology.

Theorem 2.1. Let $A=A(\alpha, \beta, 0)$ be a down-up algebra. Define

$$
s_{1}=\frac{t(2+3 t)}{1-t^{2}}, \quad s_{2}=\frac{t^{2}}{1-t^{4}},
$$

and for $n \geq 1$,

$$
f_{n}=\frac{1}{\left(1-t^{4}\right)\left(1-t^{n}\right)^{2}}, \quad g_{n}=\frac{t^{2}-t^{2 n}}{1-t^{4}}, \quad h_{n}=\frac{2 t\left(1-t^{n-1}\right)}{(1-t)\left(1-t^{n}\right)} .
$$

- If $A$ belongs to (F1), then the Hilbert series of the Hochschild homology spaces $\mathrm{HH}_{0}(A)$ and $\mathrm{HH}_{1}(A)$ are the following, while $\mathrm{HH}_{2}(A)$ and $\mathrm{HH}_{3}(A)$ vanish.

$$
H H_{0}(A)(t)=\frac{1+2 t+2 t^{2}}{1-t^{2}}, \quad H H_{1}(A)(t)=\frac{t(2+3 t)}{1-t^{2}},
$$

- If A belongs to the family (F2) and $r_{1}$ is not a root of unity, then the Hilbert series are as follows.

$$
\begin{array}{ll}
H H_{0}(A)(t)=\frac{1+2 t+2 t^{2}}{1-t^{2}}, & H H_{1}(A)(t)=\frac{t(2+3 t)}{1-t^{2}}+\frac{t^{4}}{1-t^{8}}, \\
H H_{2}(A)(t)=\frac{2 t^{4}}{1-t^{8}}, & H H_{3}(A)(t)=\frac{t^{4}}{1-t^{8}} .
\end{array}
$$

- If $A$ belongs to (F2) and $r_{1}$ is a primitive $n$-th root of unity, then

i) For $n$ even and $n \geq 4$,

$$
\begin{aligned}
& H H_{0}(A)(t)=f_{n}+h_{n}+s_{2}, \\
& H H_{1}(A)(t)=\frac{t^{4}}{\left(1-t^{4}\right)\left(1-t^{n}\right)^{2}}+2\left(f_{n}+h_{n}+s_{2}-1\right)-s_{1}, \\
& H H_{2}(A)(t)=\frac{2 t^{4}}{\left(1-t^{4}\right)\left(1-t^{n}\right)^{2}}+f_{n}+h_{n}+s_{2}-s_{1}-1, \\
& H H_{3}(A)=\frac{t^{4}}{\left(1-t^{4}\right)\left(1-t^{n}\right)^{2}} .
\end{aligned}
$$

ii) For $n$ odd and $n \geq 3$,

$$
\begin{aligned}
& H H_{0}(A)(t)=f_{n}+g_{n}+h_{n}, \\
& H H_{1}(A)(t)=\frac{t^{4}}{\left(1-t^{4}\right)\left(1-t^{n}\right)^{2}}+2\left(f_{n}+g_{n}+h_{n}-1\right)-s_{1}, \\
& H H_{2}(A)(t)=\frac{2 t^{4}}{\left(1-t^{4}\right)\left(1-t^{n}\right)^{2}}+f_{n}+g_{n}+h_{n}-s_{1}-1, \\
& H H_{3}(A)(t)=\frac{t^{4}}{\left(1-t^{4}\right)\left(1-t^{n}\right)^{2}} .
\end{aligned}
$$


iii) For $n=2$, that is $r_{1}=-1$, the Hilbert series of the Hochschild homology spaces of $A(2,-1,0)$ are

$$
\begin{array}{ll}
H H_{0}(A)(t)=\frac{1+2 t+2 t^{2}-t^{4}-2 t^{5}}{\left(1-t^{2}\right)^{2}\left(1+t^{2}\right)}, & H H_{1}(A)(t)=\frac{2 t+3 t^{2}+t^{4}-2 t^{5}}{\left(1-t^{2}\right)^{2}\left(1+t^{2}\right)}, \\
H H_{2}(A)(t)=\frac{2 t^{4}}{\left(1-t^{2}\right)^{2}\left(1+t^{2}\right)}, & H H_{3}(A)(t)=\frac{t^{4}}{1-t^{4}} .
\end{array}
$$

iv) For $n=1$, that is $r_{1}=1$, the Hilbert series of the Hochschild homology spaces of $A(2,1,0)$ are

$$
\begin{aligned}
H H_{0}(A)(t) & =\frac{1}{(1-t)^{2}}, & H H_{1}(A)(t) & =\frac{t(2-t)\left(1+t^{2}\right)}{(1-t)^{2}}, \\
H H_{2}(A)(t) & =\frac{2 t^{3}\left(1+t-t^{2}\right)}{\left(1-t^{2}\right)(1-t)}, & H H_{3}(A)(t) & =\frac{t^{4}}{1-t^{2}} .
\end{aligned}
$$

While proving this result we will also obtain the Hilbert series of the cyclic homology of $A$. Moreover, we give explicit bases of $H H_{0}(A)$ and $H H_{3}(A)$.

The computation of the Hilbert series of Hochschild cohomology spaces follows from the previous ones in the 3-Calabi-Yau case, that is, for the family (F2). However, we want to describe what happens for an algebra $A$ in (F1). No formula involving cyclic homology and the respective Hilbert series is available in this context. So, we provide explicit basis for the Hochschild cohomology spaces in this case.

Theorem 2.2 (see Section 4 for the notation). Let $A$ be a down-up algebra belonging to the family (F1). The Hilbert series of the Hochschild cohomology spaces are as follows.

$$
\begin{array}{ll}
H H^{0}(A)(t)=1, & H H^{1}(A)(t)=2, \\
H H^{2}(A)(t)=\frac{1}{t^{2}}+2+\frac{t^{2}}{1-t^{2}}, & H H^{3}(A)(t)=\frac{1}{t^{4}\left(1-t^{2}\right)} .
\end{array}
$$

Moreover,

i) $H H^{0}(A)=\mathbb{K}$,

ii) the classes of the elements $D \mid d$ and $U \mid u$ form a basis of $H H^{1}(A)$,

iii) the classes of the elements $\left\{D^{2} U\left|w_{1}^{k} d+D U^{2}\right| u w_{1}^{k}: k \geq 0\right\} \cup\left\{D^{2} U\left|u d^{2}+D U^{2}\right| u^{2} d\right\}$ form a basis of $H^{2}(A)$, and

iv) the classes of the elements $\left\{D^{2} U^{2} \mid w_{1}^{j}: j \geq 0\right.$, and $\left.j \neq 2\right\} \cup\left\{D^{2} U^{2} \mid u w_{1} d\right\}$ form a basis of $H H^{3}(A)$.

From the previous results we remark that all Hochschild homology spaces are either infinite dimensional - with finite dimensional graded components- or zero. The situation differs for Hochschild cohomology of algebras belonging to the family (F1), in which case the centre is as small as possible, that is one dimensional, and the first cohomology space has dimension 2, containing just the two obvious derivations. The fact that the second and third cohomology spaces are infinite dimensional in all cases suggests that deformations of down-up algebras are quite complicated, but having explicit bases in case (F1) indicates that the deformation theory may be nonetheless tractable. 


\section{Hochschild and cyclic homology of down-up alge- bras}

From now on we fix $\gamma=0$, and let $A=A(\alpha, \beta, 0)$ be a down-up algebra with $(\alpha, \beta)$ different from $(0,0)$. In this section we assume that the field $\mathbb{K}$ contains both roots of the polynomial $t^{2}-\alpha t-\beta$ and it is of characteristic zero.

Denote by $A(t, s)$ the Hilbert series of the bigraded algebra $A$. Consider $\mathbb{K}$ as a left $A$-module with the trivial action of $d$ and $u$. By computing the Euler-Poincaré characteristic of the exact complex $K \otimes_{A} \mathbb{K}$ we obtain

$$
A(t, s)=\frac{1}{1-t\left(s+s^{-1}\right)+t^{3}\left(s+s^{-1}\right)-t^{4}} .
$$

The Hilbert series for the usual grading is obtained by setting $s=1$ in the previous expression:

$$
A(t)=\frac{1}{\left(1-t^{2}\right)(1-t)^{2}} .
$$

Next we describe a basis of $A$ as a $\mathbb{K}$-vector space that will be useful for the computations. Denote $r_{1}$ and $r_{2}$ the roots of the polynomial $t^{2}-\alpha t-\beta$. Since $(\alpha, \beta) \neq(0,0)$ we may assume that $r_{1}$ is not zero. For $l \in\{1,2\}$ we define $w_{l}=$ $\beta u d+r_{l} d u$. It is straightforward that

$$
\begin{aligned}
& w_{l} u=r_{l} u w_{l}, \\
& d w_{l}=r_{l} w_{l} d,
\end{aligned}
$$

for $l=1,2$. Given $p \in \mathbb{Z}_{\geq 0}$, denote

$$
\phi_{p}=\sum_{i=0}^{p} r_{1}^{i} r_{2}^{p-i}=\frac{r_{1}^{p+1}-r_{2}^{p+1}}{r_{1}-r_{2}} .
$$

The last expression only holds for $r_{1} \neq r_{2}$. We set $\phi_{-1}=0$. The following identities are easily proved by induction.

Lemma 3.1. For all $k \geq 0$ the following equalities hold

$$
\begin{aligned}
& d u^{k}=\frac{\phi_{k-1}}{r_{1}} u^{k-1} w_{1}+r_{2}^{k} u^{k} d, \\
& d^{k} u=\frac{\phi_{k-1}}{r_{1}} w_{1} d^{k-1}+r_{2}^{k} u d^{k} .
\end{aligned}
$$

For the proof of the following result we refer to [20], Lemma 2.2.

Lemma 3.2. Let $l \in\{1,2\}$ and suppose $r_{l}$ is not zero. The set $\left\{u^{i} w_{l}^{j} d^{k}: i, j, k \in \mathbb{N}_{\geq 0}\right\}$ is a basis of $A$.

We denote $\overline{H C} \bullet(A), \overline{H H}_{\bullet}(A)$ and $\overline{H H}^{\bullet}(A)$ the reduced cyclic homology, the reduced Hochschild homology and the reduced Hochschild cohomology of $A$. Notice that the reduced Hochschild homology and cohomology spaces differ from the non reduced groups only in (co)homological degree zero.

Tensoring the resolution $K$ of $A$ given by (2.1) by $A$ over $A^{e}$ we obtain the following complex, whose homology is isomorphic to the Hochschild homology of $A$ :

$$
0 \rightarrow A \otimes \Omega \stackrel{d_{3}}{\rightarrow} A \otimes R \stackrel{d_{2}}{\rightarrow} A \otimes V \stackrel{d_{1}}{\rightarrow} A \rightarrow 0,
$$


where $d_{1}\left(a \otimes d+a^{\prime} \otimes u\right)=a d-d a+a^{\prime} u-u a^{\prime}$,

$$
\begin{aligned}
& d_{2}\left(a \otimes d^{2} u+a^{\prime} \otimes d u^{2}\right) \\
& =\left(d u a+u a d+u^{2} a^{\prime}-\alpha\left(u d a+a d u+u a^{\prime} u\right)-\beta\left(d a u+a u d+a^{\prime} u^{2}\right)-\gamma a\right) \otimes d \\
& +\left(a d^{2}+u a^{\prime} d+a^{\prime} d u-\alpha\left(d a d+d u a^{\prime}+a^{\prime} u d\right)-\beta\left(d^{2} a+u d a^{\prime}+d a^{\prime} u\right)-\gamma a^{\prime}\right) \otimes u,
\end{aligned}
$$

and

$$
d_{3}\left(a \otimes d^{2} u^{2}\right)=-(u a+\beta a u) \otimes d^{2} u+(a d+\beta d a) \otimes d u^{2} .
$$

Since the characteristic of $\mathbb{K}$ is zero, a theorem by T. Goodwillie (see [10], and the consequence indicated by $\mathrm{M}$. Vigué-Poirrier in [19]) tells us that, for all $i \in \mathbb{N}_{\geq 0}$ there are short exact sequences of graded vector spaces

$$
0 \rightarrow \overline{H C}_{i-1}(A) \rightarrow \overline{H H}_{i}(A) \rightarrow \overline{H C}_{i}(A) \rightarrow 0 .
$$

Since $H H_{i}(A)=0$ for all $i \geq 4$, we deduce that $\overline{H C}_{i}(A)=0$ for all $i \geq 3$.

We recall that the the Euler-Poincaré characteristic of the reduced cyclic homology $\chi_{\overline{H C} \cdot(A)}(t)$ of $A$ is defined as

$$
\chi_{\overline{H C} \cdot(A)}(t)=\sum_{p \in \mathbb{Z}}(-1)^{p} \overline{H C}_{p}(A)(t)=\overline{H C_{0}}(A)(t)-\overline{H C_{1}}(A)(t)+\overline{H C_{2}}(A)(t) .
$$

A result by K. Igusa (see [12], Thm. 3.5, Equation (16)) -and proved by different methods in an unpublished work by $\mathrm{C}$. Löfwall- tells us that it satisfies the identity $\chi_{\overline{H C} .(A)}(t)=\sum_{\ell \in \mathbb{N}} \frac{\varphi(\ell)}{\ell} \log \left(A\left(t^{\ell}\right)\right)$, where $\varphi$ is the Euler's totient function. Using that $\sum_{d \mid n} \varphi(d)=n$ for all $n \in \mathbb{N}$, we compute

$$
\sum_{\ell \in \mathbb{N}} \frac{\varphi(\ell)}{\ell} \log \left(1-t^{\ell}\right)=-\sum_{n \in \mathbb{N}}\left(\sum_{d \mid n} \varphi(d)\right) \frac{t^{n}}{n}=-\frac{t}{1-t} .
$$

Hence, using Equation 3.2 we obtain

$$
\chi_{\overline{H C} \cdot(A)}(t)=-\sum_{\ell \in \mathbb{N}} \frac{\varphi(\ell)}{\ell} \log \left(\left(1-t^{2 \ell}\right)\left(1-t^{\ell}\right)^{2}\right)=\frac{t(2+3 t)}{1-t^{2}} .
$$

Putting this all together we get

$$
\begin{aligned}
\overline{H C}_{0}(A)(t) & =\overline{H H}_{0}(A)(t) \\
\overline{H C}_{1}(A)(t) & =\overline{H H}_{0}(A)(t)+H H_{3}(A)(t)-\frac{t(2+3 t)}{1-t^{2}}, \\
\overline{H C}_{2}(A)(t) & =H H_{3}(A)(t), \\
H H_{1}(A)(t) & =2 \overline{H H}_{0}(A)(t)+H H_{3}(A)(t)-\frac{t(2+3 t)}{1-t^{2}}, \\
H H_{2}(A)(t) & =\overline{H H}_{0}(A)(t)+2 H H_{3}(A)(t)-\frac{t(2+3 t)}{1-t^{2}} .
\end{aligned}
$$

The computation of $H H_{0}(A)$ and $H H_{3}(A)$ will thus provide us the dimensions of the graded components of the other spaces.

Proposition 3.3. Let $A(\alpha, \beta, 0)$ be a down-up algebra.

1. If $A$ belongs to (F1), then the vector space $H H_{0}(A)$ has a basis formed by the classes of the elements of the set

$$
\left\{1, w_{1}^{j}, d^{j}, u^{j}: j \in \mathbb{N}\right\}
$$


2. If $A$ belongs to (F2), define $n$ as the order of $r_{1}$ if it is a root of unity and 0 otherwise. The vector space $H_{0}(A)$ has a basis formed by the classes of the following elements.

- If $n$ is even and different from 2 ,

i) $u^{i} w_{1}^{j} d^{k}$ such that $n$ divides $j-i$ and $j-k$,

ii) $u^{i}, d^{k}$ with $i, k \geq 0$ such that $n \nmid i$ and $n \nmid k$, and

iii) $w_{1}^{j}$, where $j$ is any odd number.

- If $n$ is odd and different from 1 ,

i) $u^{i} w_{1}^{j} d^{k}$ such that $n$ divides $j-i$ and $j-k$.

ii) $u^{i}, d^{k}$ with $i, k \geq 0$ such that $n \nmid i$ and $n \nmid k$, and

iii) $w_{1}^{j}$, where $j$ is odd and $j \leq n-2$.

- If $n=2$,

$$
u^{2 i} d^{2 k}, u^{2 i+1}, d^{2 k+1}, w_{1}^{2 j+1} \text { with } i, j, k \geq 0 .
$$

- If $n=1$,

$$
u^{i} d^{k} \text { with } i, j \geq 0 \text {. }
$$

Before we get to the proof of Proposition 3.3 we need some definitions and auxiliary results. Let $a=u^{i} w_{1}^{j} d^{k}$, where $i, j, k \in \mathbb{N}_{\geq 0}$. Using Lemma3.1 we deduce that

$$
a d-d a=\left(1-r_{1}^{j} r_{2}^{i}\right) u^{i} w_{1}^{j} d^{k+1}-\frac{\phi_{i-1}}{r_{1}} u^{i-1} w_{1}^{j+1} d^{k}
$$

and

$$
a u-u a=-\left(1-r_{1}^{j} r_{2}^{k}\right) u^{i+1} w_{1}^{j} d^{k}+\frac{\phi_{k-1}}{r_{1}} u^{i} w_{1}^{j+1} d^{k-1} .
$$

Define $f_{i-1, j+1, k}=a d-d a$, and $g_{i, j+1, k-1}=u a-a u$. Observe that $\operatorname{Im}\left(d_{1}\right)$ is equal to the vector space spanned by the set

$$
\left\{f_{i, j, k}: i \geq-1, j \geq 1, k \geq 0\right\} \cup\left\{g_{i, j, k}: i \geq 0, j \geq 1, k \geq-1\right\} .
$$

Let us write $t_{i}=\phi_{i} / r_{1}$ and $s_{i, j}=1-r_{1}^{j} r_{2}^{i}$. Then $f_{i, j, k}=s_{i+1, j-1} u^{i+1} w_{1}^{j-1} d^{j+1}-$ $t_{i} u^{i} w_{1}^{j} d^{k}$.

For $i, j, k$ with $t_{i} \neq 0$ and $j \geq 1$, let

$$
L_{i, j}:=\max \left\{l: \text { such that } 0 \leq l \leq j-1 \text { and } t_{i+l} \neq 0\right\}
$$

and

$$
z_{i, j, k}:=-\frac{1}{t_{i}} f_{i, j, k}-\sum_{l=1}^{L_{i, j}}\left(\frac{1}{t_{i}} \prod_{m=1}^{l} \frac{s_{i+m, j-m}}{t_{i+m}}\right) f_{i+l, j-l, k+l},
$$

where we omit the second summand whenever $L_{i, j}=0$. In order to simplify notations, let $L=L_{i, j}$. Notice that

$$
z_{i, j, k}=u^{i} w_{1}^{j} d^{k}-\left(\frac{s_{i+L+1, j-L-1}}{t_{i}} \prod_{m=1}^{L} \frac{s_{i+m, j-m}}{t_{i+m}}\right) u^{i+L+1} w_{1}^{j-L-1} d^{k+L+1}
$$

and that it belongs to $\operatorname{Im}\left(d_{1}\right)$. On the other hand, define

$$
\Gamma=\left\{(i, j, k) \in \mathbb{N}_{0}^{3}: r_{1}^{j} r_{2}^{i}=1 \text { or } k=0\right\} \cap\left\{(i, j, k) \in \mathbb{N}_{0}^{3}: r_{1}^{j} r_{2}^{k}=1 \text { or } i=0\right\} .
$$

Lemma 3.4. Let $i, j, k \geq 0$ and let $x \in \operatorname{Im}\left(d_{1}\right)$ be such that the coefficient of $u^{i} w_{1}^{j} d^{k}$ in $x$ is not zero. If $(i, j, k) \in \Gamma$, then $j \geq 1$. If in addition $n \mid i-k$, then $t_{i} \neq 0$. 
Proof. Write $x=\sum_{a, b, c \geq 0}\left(\epsilon_{a, b, c}\left[u^{a} w_{1}^{b} d^{c}, d\right]+\mu_{a, b, c}\left[u^{a} w_{1}^{b} d^{c}, u\right]\right)$. The coefficient of $u^{i} w_{1}^{j} d^{k}$ in this expression is

$$
\epsilon_{i, j, k-1} s_{i, j}-\epsilon_{i+1, j-1, k} t_{i}-\mu_{i-1, j, k} s_{k, j}+\mu_{i, j-1, k+1} t_{k},
$$

where elements with negative subindices are zero. If $(i, j, k)$ belongs to $\Gamma$, then this element is equal to $-\epsilon_{i+1, j-1, k} t_{i}+\mu_{i, j-1, k+1} t_{k}$. By hypothesis this is not zero. We deduce that $j \geq 1$. If $n \mid i-k$, then $t_{k}=t_{i}$ and the last expression is equal to $\left(-\epsilon_{i+1, j-1, k}+\mu_{i, j-1, k+1}\right) t_{i}$. Since this is not zero, we obtain $t_{i} \neq 0$.

Let $\Gamma_{0}$ be the set formed by the elements $(i, j, k) \in \Gamma$ such that

- $n \mid i-k$, and

- $j=0$ or $t_{i}=0$ or $u^{i} w_{1}^{j} d^{k} \neq z_{i, j, k}$.

Lemma 3.5. The set consisting of the classes in $H H_{0}(A)$ of the elements $u^{i} w_{1}^{j} d^{k}$ with $(i, j, k) \in \Gamma_{0}$ is linearly independent.

Proof. Let $\Gamma^{\prime} \subseteq \Gamma_{0}$ be a finite set and let $\lambda_{\gamma} \in \mathbb{K}^{\times}$, with $\gamma \in \Gamma^{\prime}$, be such that $\sum_{\gamma \in \Gamma^{\prime}} \lambda_{\gamma} u^{\gamma_{1}} w_{1}^{\gamma_{2}} d^{\gamma_{3}} \in \operatorname{Im}\left(d_{1}\right)$. We may further assume, without loss of generality, that $\sum_{\gamma \in \Gamma^{\prime}} \lambda_{\gamma} u^{\gamma_{1}} w_{1}^{\gamma_{2}} d^{\gamma_{3}}$ belongs to the subspace of $\operatorname{Im}\left(d_{1}\right)$ spanned by the homogeneous elements of special degree divisible by $n$.

It is easy to check that $f_{i, j, k}=g_{i, j, k}$ for all $i \geq 0, j \geq 1$ and $k \geq 0$ such that $n \mid i-k$. Therefore, the subspace of $\operatorname{Im}\left(d_{1}\right)$ spanned by the homogeneous elements of special degree divisible by $n$ is the $\mathbb{K}$-span of the set

$$
\left\{f_{i, j, k}: i \geq-1, j \geq 1, k \geq 0\right\} \cup\left\{g_{i, j,-1}: i \geq 0, j \geq 1\right\} .
$$

Thus,

$$
\sum_{\gamma \in \Gamma^{\prime}} \lambda_{\gamma} u^{\gamma_{1}} w_{1}^{\gamma_{2}} d^{\gamma_{3}}=\sum_{i \geq 0, j \geq 1, k \geq 0} \mu_{i, j, k} f_{i, j, k}+\sum_{j \geq 1, k \geq 0} \mu_{j, k} f_{-1, j, k}+\sum_{i \geq 0, j \geq 1} \mu_{i, j}^{\prime} g_{i, j,-1} .
$$

Let $(a, b, c)$ be an element in $\Gamma^{\prime}$ and denote $L=L_{a, b}$. By Lemma 3.4 we obtain $b \geq 1$ and $t_{a} \neq 0$. As a consequence $u^{a} w_{1}^{b} d^{c} \neq z_{a, b, c}$. This implies $s_{a+m, b-m} \neq 0$ for all $m=1, \ldots, L+1$.

Notice that $f_{-1, j, k}=\left(1-r_{1}^{j-1}\right) w_{1}^{j-1} d^{k+1}$ and $g_{i, j,-1}=\left(1-r_{1}^{j-1}\right) u^{i+1} w_{1}^{j-1}$, and that the elements $(0, j-1, k+1)$ and $(i+1, j-1,0)$ belong to $\Gamma$ if and only if $1-r_{1}^{j-1}=0$. Since $(a, b, c) \in \Gamma^{\prime} \subseteq \Gamma$, the coefficient of $u^{a} w_{1}^{b} d^{c}$ on the right hand side of the above equation is

$$
\mu_{a-1, b+1, c-1} s_{a, b}-\mu_{a, b, c} t_{a} .
$$

On the left hand side its coefficient is $\lambda_{a, b, c}$. Since $(a, b, c) \in \Gamma$, it follows that $\mu_{a-1, b+1, c-1} s_{a, b}=0$. Therefore $\mu_{a, b, c}=-\lambda_{a, b, c} t_{a}^{-1} \neq 0$. The fact that $s_{a+m, b-m} \neq 0$ for $m=1, \ldots, L+1$ implies $(a+m, b-m, c+m) \notin \Gamma$ and as a consequence the coefficient of $u^{a+m} w_{1}^{b-m} d^{c+m}$ on the left hand side of Equation 3.10 is 0, for the same values of $m$. We thus obtain

$$
\mu_{a+l, b-l, c+l}=\mu_{a+l-1, b-l+1, c+l-1} \frac{s_{a+l, b-l}}{t_{a+l}},
$$

for $1 \leq l \leq L$. We deduce $\mu_{a+L, b-L, c+L} \neq 0$. On the other hand, either $L=b-1$ or $t_{a+L+1}=0$. In either case $\mu_{a+L+1, b-L-1, c+L+1} t_{a+L+1}=0$. Looking at the coefficient of $u^{a+L+1} w_{1}^{b-L-1} d^{c+L+1}$ on both sides of (3.10) we obtain $\mu_{a+L, b-L, c+L} s_{a+L+1, b-L-1}=0$. This is a contradiction. 
Proof of Proposition 3.3 Using Equations (3.8) and (3.9) in order to obtain rewriting rules, it is clear that $H H_{0}(A)$ is generated by the classes of the elements $u^{i} w_{1}^{j} d^{k}$ with $(i, j, k) \in \Gamma$.

For an algebra $A$ in the family (F1), $n=0$ and $\Gamma$ is the set

$$
\{(0,0, k): k \geq 0\} \cup\{(i, 0,0): i \geq 0\} \cup\{(0, j, 0): j \geq 0\} .
$$

Suppose

$$
\sum_{k \geq 1} \lambda_{k} d^{k}+\sum_{i \geq 1} \mu_{i} u^{i}+\sum_{j \geq 0} \epsilon_{j} w_{1}^{j} \in \operatorname{Im}\left(d_{1}\right)
$$

for some $\lambda_{k}, \mu_{i}, \epsilon_{j} \in \mathbb{K}$. By Lemma 3.4 it follows that $\lambda_{k}=0=\mu_{i}$ for all $i, k \geq 1$ and $\epsilon_{0}=0$. Since $A$ belongs to (F1), the element $s_{m, j-m} \neq 0$ for all $j \geq 1$ and $1 \leq m \leq j$. This implies that $(0, j, 0) \in \Gamma_{0}$ for all $j \geq 1$. By Lemma 3.5 we have that $\epsilon_{j}=0$ for all $j \geq 1$. As a consequence, the classes in $H H_{0}(A)$ of the elements of the set

$$
\left\{d^{k}: k \geq 1\right\} \cup\left\{u^{i}: i \geq 1\right\} \cup\left\{w^{j}: j \geq 0\right\},
$$

form a basis and we obtain the first claim of Proposition 3.3

Let $A$ be an algebra in the family (F2) such that $r_{1}$ is different from 1 and -1 . In this case $r_{2}=r_{1}^{-1}$ and $n$ is different from 1 and 2. Here $\Gamma$ is the set of elements $(i, j, k) \in \mathbb{N}_{0}^{3}$ satisfying any of the following properties.
1. $n \mid j-i$ and $n \mid j-k$.
4. $i=k=0$ and $n \nmid j$.
2. $i=j=0$ and $n \nmid k$.
5. $i=0, n \mid j, n \nmid k$ and $j \geq 1$.
3. $j=k=0$ and $n \nmid i$.
6. $k=0, n \mid j, n \nmid i$ and $j \geq 1$.

Let us see that the elements $u^{i} w_{1}^{j} d^{k}$ with $(i, j, k)$ of types 5 and 6 belong to $\operatorname{Im}\left(d_{1}\right)$. Let $(i, j, k)$ be of type 5 . We have $w_{1}^{j} d^{k}=r_{1}\left(1-r_{1}^{-2}\right) u w_{1}^{j-1} d^{k+1}-r_{1} f_{0, j, k}$ and

$$
u w_{1}^{j-1} d^{k+1}=\frac{\phi_{k} f_{0, j, k}-g_{0, j, k}}{r_{1}^{k}-1} .
$$

We deduce that the element $w_{1}^{j} d^{k}$ belongs to $\operatorname{Im}\left(d_{1}\right)$. The case where $(i, j, k)$ is of type 6 is similar. As a consequence, the homology space $H H_{0}(A)$ is generated by the classes of elements $u^{i} w_{1}^{j} d^{k}$ with $(i, j, k)$ of type $1,2,3$ or 4 . Observe that if $(i, j, k) \in \Gamma$ is not of type 5 or 6 , then either $j=0$ or $n \mid i-k$. If $j \geq 1$ and $(i, j, k) \notin \Gamma_{0}$, then $u^{i} w_{1}^{j} d^{k}=z_{i, j, k} \in \operatorname{Im}\left(d_{1}\right)$. Thus, we can remove it from our set of generators. Using Lemmas 3.4 and 3.5 we deduce that the set of classes of elements $u^{i} w_{1}^{j} d^{k}$ with $(i, j, k)$ in

$$
\Gamma_{1}:=\left\{(i, j, k) \in \Gamma:(i, j, k) \text { is of type } 1,2,3 \text { or } 4 \text {, and } j=0 \text { or }(i, j, k) \in \Gamma_{0}\right\},
$$

is a basis of $H H_{0}(A)$. Now we describe the set $\Gamma_{1}$.

Let $(i, j, k)$ be of type 1 with $j \geq 1$ and $t_{i} \neq 0$. Denote $L=L_{i, j}$. We have $t_{i+l}=0$ if and only if $n \mid 2(i+l+1)$. On the other hand $s_{i+m, j-m}=0$ if and only if $n \mid 2 m$. In particular $s_{i, j}=0$. If $n$ is odd, then there exists $0 \leq l \leq n-1$ such that $n \mid 2(i+l+1)$, which implies $L \leq n-2$. Similarly, in case $n$ is even, we obtain $L \leq n / 2-2$. In either case $s_{m, j-m} \neq 0$ for all $m=1, \ldots, L+1$ and as a consequence $u^{i} w_{1}^{j} z^{k} \neq z_{i, j, k}$. This implies $(i, j, k) \in \Gamma_{1}$ for all $(i, j, k)$ of type 1 .

Suppose $n$ is even. Let $(0, j, 0)$ be of type 4 . Since $n \nmid j$ we have $j \geq 1$. Let $L=L_{0, j}$. The element $t_{l}$ is zero if and only if $n \mid 2(l+1)$. Therefore $L=\min \{n / 2-$ $2, j-1\}$. On the other hand $s_{m, j-m}$ is zero if and only if $n \mid j-2 m$. If $j$ is odd, then this last condition is never satisfied and $s_{m, j-m} \neq 0$ for all $m$, from where 
we deduce $w^{j} \neq z_{0, j, 0}$ and $(0, j, 0) \in \Gamma_{1}$. Suppose $j$ is even. If $L=j-1$, then $s_{m, j-m}=0$ for $m=j / 2$ and $j / 2<L+1$, which implies $w^{j}=z_{0, j, 0}$. If $L=n / 2-2$, so there exists $1 \leq m \leq n / 2-1=L+1$ such that $n / 2$ divides $j / 2-m$. This implies $s_{m, j-m}=0$ and $w^{j}=z_{0, j, 0}$. We conclude that an element $(0, j, 0)$ of type 4 belongs to $\Gamma_{1}$ if and only if it $j$ is odd. We have proven the first part of the second claim of Proposition 3.3

Suppose $n$ is odd and let $(0, j, 0)$ be of type 4 . Set $L=L_{0, j}$. In this case $t_{l}=0$ if and only if $n \mid l+1$. We deduce $L=\min \{j-1, n-2\}$. On the other hand, $s_{m, j-m}=0$ if and only if $n \mid j-2 m$. Since 2 is invertible modulo $n$ and $n \nmid j$, this condition is always satisfied for some $1 \leq m \leq n-1$. Therefore, if $j \geq n-1$, we obtain $w^{j}=z_{0, j, 0}$ and $(0, j, 0) \notin \Gamma_{1}$. Suppose $j \leq n-2$. We have $L=j-1$. The absolute value of $j-2 m$ is positive and strictly less than $n$ for all $m=1, \ldots, j$. Thus, if $1 \leq m \leq n-1$ is such that $n \mid j-2 m$, we get $m \geq j+1$. This implies

$$
\max \left\{m \geq 1: s_{m, j-m} \neq 0\right\} \geq j+1>L+1 .
$$

As a consequence $w^{j} \neq z_{0, j, 0}$ and $(0, j, 0) \in \Gamma_{1}$. This proves the second part of the second claim Proposition 3.3 .

The proof of the cases where $n=1$ or $n=2$ is similar.

We will now describe $H_{3}(A)$. The result depends heavily on whether the algebra belongs to (F1) or to (F2). In the first case $H_{3}(A)$ annihilates, while for (F2) the dimension is always infinite, for which the basis differs considerably in the root of unity case.

Proposition 3.6. Let $A=A(\alpha, \beta, 0)$ be a down-up algebra.

1. If $A$ belongs to (F1), then $\mathrm{HH}_{3}(A)$ vanishes.

2. If A belongs to (F2), the Hochschild homology group $\mathrm{HH}_{3}(A)$ has a basis formed by the classes of the elements of the set

- $\left\{w_{1}^{2 i} w_{2}^{2 i} \mid d^{2} u^{2}: i \geq 0\right\}$ if $r_{1}$ is not a root of unity,

- $\left\{w_{1}^{i} w_{2}^{j} u^{n k} d^{n l}\left|d^{2} u^{2}: n\right| i-j\right.$ and $\left.k l=0\right\}$ if $r_{1}$ is a primitive $n$-th root of unity with $n \geq 3$,

- $\left\{w_{1}^{2 i} \mid d^{2} u^{2}: i \geq 0\right\}$ if $r_{1}=-1$,

- $\left\{w_{1}^{i} \mid d^{2} u^{2}: i \geq 0\right\}$ if $r_{1}=1$.

Proof. Let $v \in \operatorname{Ker} d_{3}$. Since the differentials respect the bidegree, we may assume $v$ is homogeneuos of bidegree $(s, t)$. Let $l=(s+t) / 2$. An element $u^{i} w_{1}^{j} d^{k}$ homogeneous of bidegree $(s, t)$ satisfies $j=l-i \geq 0$ and $k=i-t \geq 0$. As a consequence, we deduce $l \geq 0$ and $t \leq l$. Set

$$
v=\sum_{i} c_{i} u^{i} w_{1}^{l-i} d^{i-t},
$$

where $c_{i}$ vanishes either when $i<0, l-i<0$ or when $i-t<0$. Using the formulas in Fact 3.1 we obtain the following equality.

$$
\begin{aligned}
& d_{3}\left(v \mid d^{2} u^{2}\right) \\
& \quad=-\left(\sum c_{i}\left(\left(1+\beta r_{1}^{l-i} r_{2}^{i-t}\right) u^{i+1} w_{1}^{l-i} d^{i-t}+\beta \frac{\phi_{i-t-1}}{r_{1}} u^{i} w_{1}^{l-i+1} d^{i-t-1}\right)\right) \mid d^{2} u \\
& \quad+\left(\sum c_{i}\left(\left(1+\beta r_{1}^{l-i} r_{2}^{i}\right) u^{i} w_{1}^{l-i} d^{i-t+1}+\beta \frac{\phi_{i-1}}{r_{1}} u^{i-1} w_{1}^{l-i+1} d^{i-t}\right)\right) \mid d u^{2} .
\end{aligned}
$$


The condition $d_{3}\left(v \mid d^{2} u^{2}\right)=0$ implies the vanishing of each summand separately. By looking at the coefficient of $u^{a+1} w_{1}^{l-a} d^{a-t}$ in the first constraint and at the coefficient of $u^{a} w_{1}^{l-a} d^{a-t+1}$ in the second constraint, we obtain the following identities. For all $a \geq 0$,

$$
\begin{aligned}
& 0=c_{a}\left(1+\beta r_{1}^{l-a} r_{2}^{a-t}\right)+c_{a+1} \beta \frac{\phi_{a-t}}{r_{1}}, \\
& 0=c_{a}\left(1+\beta r_{1}^{l-a} r_{2}^{a}\right)+c_{a+1} \beta \frac{\phi_{a}}{r_{1}} .
\end{aligned}
$$

Suppose $A$ belongs to (F1). The first equality implies that $c_{a}=\mu_{a} c_{a+1}$, where $\mu_{a}=\beta \phi_{a-t}\left(r_{1}\left(1+\beta r_{1}^{l-a} r_{2}^{a-t}\right)\right)^{-1}$. Since $c_{a}=0$ for all $a>l$, we deduce $c_{a}=0$ for all $a$. As a consequence, $\mathrm{HH}_{3}(A)=0$.

Suppose now that $A$ belongs to (F2) and $r_{1}$ is not a root of unity. Using the fact that $r_{2}=r_{1}^{-1}$, the equalities above are

$$
\begin{aligned}
& 0=c_{a}\left(1-r_{1}^{l-2 a+t}\right)-c_{a+1} \frac{\phi_{a-t}}{r_{1}}, \\
& 0=c_{a}\left(1-r_{1}^{l-2 a}\right)-c_{a+1} \frac{\phi_{a}}{r_{1}} .
\end{aligned}
$$

If $l$ is odd, the second equality and an argument similar to the case (F1) show that $c_{a}=0$ for all $a$, and so $v=0$. Suppose $l$ is even. We may use the second equation for $a$ ranging from $l$ to $l / 2+1$ and the previous argument to deduce $c_{a}=0$ for $a \in\{l / 2+1, \cdots, l\}$. Replacing $a=l / 2$ in the first equation we obtain $c_{l / 2}\left(1-r_{1}^{t}\right)=0$. If $t \neq 0$, then $c_{l / 2}=0$, and the first equation for values of $a$ ranging from $l / 2-1$ to 0 proves that $c_{a}=0$ for all $a$ and therefore $v=0$. If $t=0$, then the same argument proves that there exists $\mu_{a} \in k$ such that $c_{a}=\mu_{a} c_{l / 2}$ for all $a$. Observe that in this case $l$ is even and $t=0$, which implies $s=4 k$ for some $k \in \mathbb{Z}$. As a consequence, the homogeneous component of bidegree $(s, t)$ of Ker $d_{3}$ is trivial if $(s, t) \neq(4 k, 0)$ for some $k \in \mathbb{Z}$, and in case $(s, t)=(4 k, 0)$, it is one dimensional. Using (3.3) it is easy to see that the element $w_{1}^{2 k} w_{2}^{2 k}$ belongs to the homogeneous component of bidegree $(4 k, 0)$.

The case where $A$ belongs to (F2) and $r_{1}$ is a root of unity follows from [14], Lemma 2.0.1, Theorems 4.0.3 and 4.0.4, together with the fact that $A$ is 3-CalabiYau, so $H H_{3}(A)[4] \cong H H^{0}(A)$. (3.7).

Theorem 2.1 follows from Proposition 3.3. Proposition 3.6 and the identities in

\section{Hochschild cohomology}

As we mentioned in Section 3 , if $A$ belongs to (F2), then it is 3-Calabi-Yau and the dimension of the Hochschild cohomology spaces can be deduced from Theorem 2.1. since $H H^{i}(A)$ is isomorphic to $H H_{3-i}(A)[4]$ for all $i \in\{0,1,2,3\}$. We use again the minimal resolution of $A$ as $A$-bimodule to obtain the following complex whose homology is isomorphic to the Hochschild cohomology of $A$.

$$
0 \rightarrow A \stackrel{d_{0}^{*}}{\rightarrow} V^{*} \otimes A \stackrel{d_{1}^{*}}{\rightarrow} R^{*} \otimes A \stackrel{d_{2}^{*}}{\rightarrow} \Omega^{*} \otimes A \rightarrow 0,
$$

where $V^{*}$ is the dual space of $V$ spanned by the basis $\{U, D\}$, and similarly for $R^{*}$ and $\Omega^{*}$. The differentials are given by

$$
\begin{aligned}
& d_{0}^{*}(a)=U|(u a-a u)+D|(d a-a d), \\
& d_{1}^{*}(x)=D^{2} U \otimes \Delta_{1}(x)+D U^{2} \otimes \Delta_{2}(x),
\end{aligned}
$$


where, for $x=U \otimes a+D \otimes a^{\prime}$,

$$
\begin{aligned}
& \Delta_{1}(x)=d^{2} a+a^{\prime} d u+d a^{\prime} u-\alpha\left(d a d+a^{\prime} u d+d u a^{\prime}\right)-\beta\left(a d^{2}+u a^{\prime} d+u d a^{\prime}\right)-\gamma a^{\prime}, \\
& \Delta_{2}(x)=d a u+d u a+a^{\prime} u^{2}-\alpha\left(a d u+u d a+u a^{\prime} u\right)-\beta\left(a u d+u a d+u^{2} a^{\prime}\right)-\gamma a,
\end{aligned}
$$

and

$$
d_{2}^{*}\left(D^{2} U \otimes a+D U^{2} \otimes a^{\prime}\right)=D^{2} U^{2} \otimes\left(d a^{\prime}+\beta a^{\prime} d-a u-\beta u a\right) .
$$

Clearly $H H^{i}(A)=0$ for all $i \geq 4$. From now on, assume that $A$ belongs to (F1). Note that by defining $\operatorname{bideg}(U)=(-1,-1)$ and $\operatorname{bideg}(D)=(1,-1)$, the differentials of the complex (4.1) are of bidegree zero. We recall that $H H^{0}(A)$ is the center $\mathscr{Z}(A)$ of the algebra $A$.

Proposition 4.1. Let $A=A(\alpha, \beta, 0)$ be a down-up algebra of the family (F1). The cohomology space $H H^{0}(A)$ is $\mathbb{K} .1_{A}$.

Proof. It is clear that $\mathbb{K} \cdot 1_{A} \subseteq \mathscr{Z}(A)$. We shall prove the other inclusion. Let

$$
a=\sum c_{i j k} u^{i} w_{1}^{j} d^{k}
$$

where the sum is indexed over all integers $i, j, k \in \mathbb{N}_{\geq 0}$, the support is finite and $c_{i j k} \in \mathbb{K}$. Let us suppose that $a \in \mathscr{Z}(A)$, so in particular $u a-a u=0$. It suffices to prove that $c_{i j k}=0$ for all $(i, j, k) \in \mathbb{N}_{\geq 0}^{3} \backslash\{(0,0,0)\}$. Using the identities (3.3) we get that

$$
a d-d a=\sum c_{i j k}\left(\left(1-r_{1}^{j} r_{2}^{i}\right) u^{i} w_{1}^{j} d^{k+1}-\frac{\phi_{i-1}}{r_{1}} u^{i-1} w_{1}^{j+1} d^{k}\right)
$$

and

$$
a u-u a=-\sum c_{i j k}\left(\left(1-r_{1}^{j} r_{2}^{k}\right) u^{i+1} w_{1}^{j} d^{k}-\frac{\phi_{k-1}}{r_{1}} u^{i} w_{1}^{j+1} d^{k-1}\right) .
$$

Since $a \in \mathscr{Z}(A)$, both two expressions vanish. By regarding the total coefficient of the monomial $u^{i_{0}+1} d^{k_{0}}$ on the right hand side of (4.5) we get that $c_{i_{0}, 0, k_{0}}=0$ if $k_{0} \neq 0$, since in this case $\left(1-r_{2}^{k_{0}}\right) \neq 0$. Analogously, since the total coefficient of the monomial $u^{i_{0}} d^{k_{0}+1}$ on the right hand side of (4.4) vanishes, we see that $c_{i_{0}, 0, k_{0}}=0$ if $i_{0} \neq 0$, since in this case $\left(1-r_{2}^{i_{0}}\right) \neq 0$. As a consequence, we conclude that $c_{i, 0, k}=0$, for all $(i, k) \in \mathbb{N}_{0}^{2} \backslash\{(0,0)\}$.

The vanishing of the coefficient of the monomial $u^{i_{0}+1} w^{j_{0}} d^{k_{0}}$ on the right hand side of (4.5), for $j_{0}>0$, implies that

$$
c_{i_{0}, j_{0}, k_{0}}=-\frac{c_{i_{0}+1, j_{0}-1, k_{0}+1}}{\left(1-r_{2}^{k_{0}} r_{1}^{j_{0}}\right)} .
$$

Note that the hypothesis of genericity implies that the denominator never vanishes. Iterating this identity we obtain that $c_{i j k}$ is proportional to $c_{i+j, 0, k+j}$, which vanishes if $(i, j, k) \neq(0,0,0)$, and so $c_{i j k}=0$ for all $(i, j, k) \in \mathbb{N}_{\geq 0}^{3} \backslash\{(0,0,0)\}$, thus proving the proposition.

Proposition 4.2. Let $A=A(\alpha, \beta, 0)$ be a down-up algebra of the family (F1). The cohomology space $H H^{3}(A)$ is isomorphic to the $\mathbb{K}$-vector space spanned by the classes of the elements of the set

$$
\left\{D^{2} U^{2} \mid w_{1}^{j}: j \geq 0 \text { and } j \neq 2\right\} \cup\left\{D^{2} U^{2} \mid u w_{1} d\right\} .
$$

Proof. Identifying the space $\Omega^{*} \otimes A$ with $A$, the cohomology space $H H^{3}(A)$ is $A / S$, where $S$ is the $\mathbb{K}$-vector space $\left\{d a+\beta a d+a^{\prime} u+\beta u a^{\prime}: a, a^{\prime} \in A\right\}$. Denote by 
$\pi: A \rightarrow H H^{3}(A)$ the canonical projection. If $v$ is an element of $H H^{3}(A)$, denote by $\mathbb{K} v$ the $\mathbb{K}$-vector space spanned by it.

We shall prove that the classes of $\left\{w_{1}^{j}: j \geq 2\right.$ and $\left.j \neq 2\right\} \cup\left\{u w_{1} d\right\}$ in the cohomology space $H H^{3}(A)$ form a basis. Let $a=u^{i} w_{1}^{j} d^{k}$, where $i, j, k \in \mathbb{N}_{\geq 0}$. It is straightforward to compute

$$
d a+\beta a d=\left(\beta+r_{1}^{j} r_{2}^{i}\right) u^{i} w_{1}^{j} d^{k+1}+\frac{\phi_{i-1}\left(r_{1}, r_{2}\right)}{r_{1}} u^{i-1} w_{1}^{j+1} d^{k},
$$

and

$$
a u+\beta u a=\left(\beta+r_{1}^{j} r_{2}^{k}\right) u^{i+1} w_{1}^{j} d^{k}+\beta \frac{\phi_{k-1}\left(r_{1}, r_{2}\right)}{r_{1}} u^{i} w_{1}^{j+1} d^{k-1} .
$$

The hypothesis of genericity implies that $\beta+r_{1}^{j} r_{2}^{l}=0$ if and only if $(i, l)=(1,1)$. Note that the first coefficient on the right hand side of the above equations is of this form. Setting $i=0$ in (4.6) and $k=0$ in (4.7) we obtain that $w_{1}^{j} d^{l}$ and $u^{l} w_{1}^{j}$ belong to $S$ for all $j \geq 0$ and $l \geq 1$. Let $i, j, k \geq 0$. Equation (4.6) implies that $\pi\left(u^{i} w_{1}^{j} d^{k}\right) \in$ $\mathbb{K} \pi\left(u^{i-1} w_{1}^{j+1} d^{k-1}\right)$ for all $k \geq 1$ and $(i, j) \neq(1,1)$. Suppose $(i, j) \notin\{(1,1),(2,0)\}$. By a repeated use of (4.6) and the remarks above, we conclude that $u^{i} w_{1}^{j} d^{k}$ lies in $S$ if $i \neq k$, and we also deduce that $\pi\left(u^{i} w_{1}^{j} d^{i}\right) \in K \pi\left(w_{1}^{i+j}\right)$. By a similar argument using (4.7) we obtain that in case $(i, j) \in\{(1,1),(2,0)\}$, the element $\pi\left(u^{i} w_{1}^{j} d^{k}\right)$ belongs to $\mathbb{K} \pi\left(u w_{1} d\right)$ for all $k$. As a consequence, the set $\left\{\pi\left(w_{1}^{j}\right): j \geq 2\right\} \cup\left\{\pi\left(u^{2} d^{2}\right)\right\}$ generates $H H^{3}(A)$ as a $\mathbb{K}$-vector space. On the other hand, (4.6) tells us that the element $\pi\left(w_{1}^{2}\right)$ vanishes for $(i, j, k)=(1,1,0)$.

Let us see that the set $\left\{\pi\left(w_{1}^{j}\right): j \geq 2\right\} \cup\left\{\pi\left(u^{2} d^{2}\right)\right\}$ is linearly independent. Suppose there exist elements $\lambda_{j} \in \mathbb{K}$, with $j \geq 0$, and $a, a^{\prime} \in A$, such that

$$
\sum_{j \neq 2} \lambda_{j} w_{1}^{j}+\lambda_{2} u w_{1} d=d a+\beta a d+a u+\beta u a .
$$

Let $l \geq 0$. By looking at the homogeneous component of bidegree $(2 l, 0)$ in the equation above, we deduce that there exist $\epsilon_{k, j} \in \mathbb{K}$ such that

$$
\begin{array}{rlrl}
\lambda_{l} w_{1}^{l} & =\sum_{j+k=l-1} \epsilon_{j, k}\left(f_{j, k}\right), & & \text { if } l \neq 2, \\
\lambda_{2} u w_{1} d & =\sum_{j+k=1} \epsilon_{j, k}\left(f_{j, k}\right), & \text { if } l=2,
\end{array}
$$

where $f_{j, k}=\left(u^{k+1} w_{1}^{j} d^{k}\right) d+\beta d\left(u^{k+1} w_{1}^{j} d^{k}\right)$ for all $k, j \geq 0$. It is easy to see that these equations imply $\lambda_{j}=0$ for all $j \geq 0$.

Proposition 4.3. Let $A=A(\alpha, \beta, 0)$ be a down-up algebra of the family (F1). The cohomology space $H H^{1}(A)$ is 2-dimensional and it is spanned by the classes of $\{D|d, U| u\}$.

We shall first prove the following intermediate result.

Lemma 4.4. Under the same assumptions of the proposition, the $\mathbb{K}$-vector space $\left(V^{*} \otimes\right.$ A) $/ \operatorname{Im}\left(d_{0}^{*}\right)$ is spanned by the classes of the elements of the set

$$
\mathscr{S}=\left\{U\left|u^{l}, D\right| d^{l}: l \in \mathbb{N}\right\} \cup\left\{U \mid u^{i} w_{1}^{j} d^{k}: i-k \leq 0\right\} \cup\left\{D \mid u^{i} w_{1}^{j} d^{k}: i-k \geq-1\right\} .
$$

Proof. Note that there is some redundancy in our description of $\mathscr{S}$, since for example $D \mid d$ belongs both to the first and to the third subset of the union. If $a=u^{i} w_{1}^{j} d^{k}$, then $d_{0}^{*}(a)$ equals

$$
\begin{aligned}
& U \mid\left(\left(1-r_{1}^{j} r_{2}^{k}\right) u^{i+1} w_{1}^{j} d^{k}-\frac{\phi_{k-1}\left(r_{1}, r_{2}\right)}{r_{1}} u^{i} w_{1}^{j+1} d^{k-1}\right) \\
&-D \mid\left(\left(1-r_{1}^{j} r_{2}^{i}\right) u^{i} w_{1}^{j} d^{k+1}-\frac{\phi_{i-1}\left(r_{1}, r_{2}\right)}{r_{1}} u^{i-1} w_{1}^{j+1} d^{k}\right) .
\end{aligned}
$$


Suppose $x_{a, b, c}=U \mid u^{a} w_{1}^{b} d^{c} \notin \mathscr{S}$, then $b+c>0$ and $a-c>0$. We shall show that $x_{a, b, c}$ belongs to the subspace spanned by $\mathscr{S}$ and $\operatorname{Im}\left(d_{0}^{*}\right)$. In order to do so, first notice that Equation (4.8) for $i=a-1, j=b$ and $k=0$ tells us that $x_{a, b, 0}=U \mid u^{a} w_{1}^{b}$ belongs to the subspace spanned by $\mathscr{S}$ and $\operatorname{Im}\left(d_{0}^{*}\right)$, since for $b>0$ the coefficient of $x_{a, b, 0}$ in (4.8) is nonzero by the hypothesis of genericity. Moreover, Equation (4.8) for $i=a-1, j=b$ and $k=c$ tells us that $x_{a, b, c}=U \mid u^{a} w_{1}^{b} d^{c}$ belongs to the subspace spanned by $x_{a-1, b+1, c-1}$, the set $\mathscr{S}$ and $\operatorname{Im}\left(d_{0}^{*}\right)$, because, for $b+c>0$, the coefficient of $x_{a, b, c}$ in (4.8) is nonzero due to the hypothesis of genericity. By a recursive argument we prove that $x_{a, b, c}=U \mid u^{a} w_{1}^{b} d^{c}$ belongs to the subspace spanned by $\mathscr{S}$ and $\operatorname{Im}\left(d_{0}^{*}\right)$.

Analogously, let $x_{a^{\prime}, b^{\prime}, c^{\prime}}^{\prime}=D \mid u^{a^{\prime}} w_{1}^{b^{\prime}} d^{c^{\prime}} \notin \mathscr{S}$. Thus, $a^{\prime}+b^{\prime}>0$ and $a^{\prime}-c^{\prime}<-1$. We claim that $x_{a^{\prime}, b^{\prime}, c^{\prime}}^{\prime}$ belongs to the subspace spanned by the set $\mathscr{S}$ and $\operatorname{Im}\left(d_{0}^{*}\right)$. Indeed, first notice that Equation (4.8) for $i=0, j=b^{\prime}$ and $k=c^{\prime}-1$ implies that $x_{0, b^{\prime}, c^{\prime}}^{\prime}=D \mid w_{1}^{b^{\prime}} d^{c^{\prime}}$ belongs to the subspace spanned by $\mathscr{S}$ and $\operatorname{Im}\left(d_{0}^{*}\right)$, since for $b>0$ the coefficient of $x_{0, b^{\prime}, c^{\prime}}^{\prime}$ in (4.8) is nonzero by the hypothesis of genericity. Furthermore, Equation (4.8) for $i=a^{\prime}, j=b^{\prime}$ and $k=c^{\prime}-1$ implies that $x_{a^{\prime}, b^{\prime}, c^{\prime}}^{\prime}=D \mid u^{a^{\prime}} w_{1}^{b^{\prime}} d^{c^{\prime}}$ belongs to the subspace spanned by $x_{a^{\prime}-1, b^{\prime}+1, c^{\prime}-1}^{\prime}$, the set $\mathscr{S}$ and $\operatorname{Im}\left(d_{0}^{*}\right)$, using that for $b^{\prime}+c^{\prime}>0$ the coefficient of $x_{a^{\prime}, b^{\prime}, c^{\prime}}^{\prime}$ in 4.8 is nonzero by the hypothesis of genericity, for $b^{\prime}+c^{\prime}>0$. A recursive argument allows us to conclude that $x_{a^{\prime}, b^{\prime}, c^{\prime}}^{\prime}=D \mid u^{a} w_{1}^{b} d^{c^{\prime}}$ belongs to the subspace spanned by $\mathscr{S}$ and $\operatorname{Im}\left(d_{0}^{*}\right)$.

Since (4.1) is a complex, the differential $d_{1}^{*}$ trivially induces a map $\bar{d}_{1}^{*}$ from $\left(V^{*} \otimes A\right) / \operatorname{Im}\left(d_{0}^{*}\right)$ to $R^{*} \otimes A$, whose kernel is the Hochschild cohomology space $H H^{1}(A)$. It is easy to prove that the classes of $U \mid u$ and $D \mid d$ belong to the kernel of $\bar{d}_{1}^{*}$, and that they are linearly independent, since the intersection between the $\mathbb{K}$ vector subspace of $V^{*} \otimes A$ spanned by $U \mid u$ and $D \mid d$ and $\operatorname{Im}\left(d_{0}^{*}\right)$ is trivial, by degree reasons. In order to complete the proof of Proposition 4.3 it suffices thus to prove the following result.

Lemma 4.5. Assume $A$ is a down-up algebra of the family (F1). Define $W$ to be the $\mathbb{K}$ vector subspace of $\left(V^{*} \otimes A\right) / \operatorname{Im}\left(d_{0}^{*}\right)$ spanned by the classes of the elements of the family $\mathscr{S}^{\prime}$ given by

$$
\left\{U\left|u^{l}, D\right| d^{l}: l \in \mathbb{N}_{\geq 2}\right\} \cup\left\{U \mid u^{i} w_{1}^{j} d^{k}: i-k \leq 0\right\} \cup\left\{D \mid u^{i} w_{1}^{j} d^{k}: i-k \geq-1\right\} \backslash\{D \mid d\} .
$$

The intersection $W \cap \operatorname{Ker}\left(\bar{d}_{1}^{*}\right)$ is trivial.

Proof. Let $x$ be an element of $\left(V^{*} \otimes A\right)$ given by a finite linear combination of the form

$$
x=\underbrace{\sum_{i-k \leq 0} c_{i, j, k} U \mid u^{i} w_{1}^{j} d^{k}}_{x_{U}}+\underbrace{\sum_{i^{\prime}-k^{\prime} \geq-1} c_{i^{\prime}, j^{\prime}, k^{\prime}}^{\prime} D \mid u^{i^{\prime}} w_{1}^{j^{\prime}} d^{k^{\prime}}}_{x_{D}}+\underbrace{\sum_{l \geq 2} a_{l} U \mid u^{l}}_{x_{U}^{\prime}}+\underbrace{\sum_{l^{\prime} \geq 2} a_{l^{\prime}}^{\prime} D \mid d^{l^{\prime}}}_{x_{D}^{\prime}},
$$

where we exclude the case $\left(i^{\prime}, j^{\prime}, k^{\prime}\right)=(0,0,1)$ in the second sum. Since the image under $\bar{d}_{1}^{*}$ of the class of $x$ in $\left(V^{*} \otimes A\right) / \operatorname{Im}\left(d_{0}^{*}\right)$ coincides with the image under $d_{1}^{*}$ of $x$, it suffices to prove that the vanishing of this last image implies that that the class of $x$ in $\left(V^{*} \otimes A\right) / \operatorname{Im}\left(d_{0}^{*}\right)$ vanishes. Without loss of generality we may take $x$ homogeneous for the bigrading, since $d_{1}^{*}$ is homogeneous of bidegree zero. Being homogeneous for the special degree implies that either $x=x_{U}+x_{D}^{\prime}$ or $x=x_{D}+x_{U}^{\prime}$, while being homogeneous for the usual degree restricts $x$ to one of the following cases:

(i) $x=x_{U}$ such that $\operatorname{deg}\left(x_{U}\right)+\mathrm{s}-\operatorname{deg}\left(x_{U}\right) \neq 0$; 
(ii) $x=c_{0,1, k-1} U\left|w d^{k-1}+c_{1,0, k} U\right| u d^{k}+a_{k+1}^{\prime} D \mid d^{k+1}$, for $k \geq 1$;

(iii) $x=x_{D}$ such that $\operatorname{deg}\left(x_{D}\right) \neq \operatorname{s}-\operatorname{deg}\left(x_{D}\right)$;

(iv) $x=c_{i-1,1,0}^{\prime} D\left|u^{i-1} w+c_{i, 0,1}^{\prime} D\right| u^{i} d+a_{i+1} U \mid u^{i+1}$, for $i \geq 1$.

Let us first consider case (iii). By definition of $d_{1}^{*}$, we write $d_{1}^{*}(x)=D^{2} U \mid \Delta_{1}(x)+$ $D U^{2} \mid \Delta_{2}(x)$. An explicit computation using formulas given in Fact 3 .1 leads to

$$
\begin{aligned}
\Delta_{2}(x)=\sum_{i^{\prime}-k^{\prime} \geq-1} c_{i^{\prime}, j^{\prime}, k^{\prime}} & \left(\frac{\phi_{k^{\prime}-1}\left(r_{1}, r_{2}\right) \phi_{k^{\prime}-2}\left(r_{1}, r_{2}\right)}{r_{1}^{2}} u^{i^{\prime}} w_{1}^{j^{\prime}+2} d^{k^{\prime}-2}\right. \\
& +\frac{\alpha \phi_{k^{\prime}-1}\left(r_{1}, r_{2}\right)}{r_{1}}\left(r_{1}^{j^{\prime}} r_{2}^{k^{\prime}-1}-1\right) u^{i^{\prime}+1} w_{1}^{j^{\prime}+1} d^{k^{\prime}-1} \\
& \left.+\left(r_{1}^{2 j^{\prime}} r_{2}^{2 k^{\prime}}-\alpha r_{1}^{j^{\prime}} r_{2}^{k^{\prime}}-\beta\right) u^{i^{\prime}+2} w_{1}^{j^{\prime}} d^{k^{\prime}}\right)
\end{aligned}
$$

and the coefficient of the monomial $u^{a} w_{1}^{b} d^{c}$ (where $a, b, c \geq 0$ ) is thus

$$
\begin{aligned}
& \frac{\phi_{c+1}\left(r_{1}, r_{2}\right) \phi_{c}\left(r_{1}, r_{2}\right)}{r_{1}^{2}} c_{a, b-2, c+2} \\
& \quad+\frac{\alpha \phi_{c}\left(r_{1}, r_{2}\right)}{r_{1}}\left(r_{1}^{b-1} r_{2}^{c}-1\right) c_{a-1, b-1, c+1}+\left(r_{1}^{2 b} r_{2}^{2 c}-\alpha r_{1}^{b} r_{2}^{c}-\beta\right) c_{a-2, b, c} .
\end{aligned}
$$

The fact that $x$ belongs to the kernel of $d_{1}^{*}$ implies the vanishing of the previous expression. In particular, we see that

$$
\begin{aligned}
& c_{a, 0, c}=0 \text { for all } c \neq 1, \\
& c_{a, 1, c}=0 \text { for all } c \neq 0, \text { and } \\
& c_{a, b, c}=0 \text { for all } b \geq 2 .
\end{aligned}
$$

Condition $\operatorname{deg}\left(x_{D}\right) \neq \mathrm{s}-\operatorname{deg}\left(x_{D}\right)$ implies that $c_{a, 0,1}=0$ and $c_{a, 1,0}=0$ for all $a$. As a consequence, $x_{D}$ vanishes in $\left(V^{*} \otimes A\right)$. Case (i) is handled mutatis mutandi.

Let us now treat case (iv), where

$$
x=c_{i-1,1,0}^{\prime} D\left|u^{i-1} w+c_{i, 0,1}^{\prime} D\right| u^{i} d+a_{i+1} U \mid u^{i+1},
$$

for some $i \geq 1$. We write again $d_{1}^{*}(x)=D^{2} U\left|\Delta_{1}(x)+D U^{2}\right| \Delta_{2}(x)$. Using the computations of the previous paragraph we see that $\Delta_{2}\left(D \mid u^{i-1} w\right)$ and $\Delta_{2}\left(D \mid u^{i} d\right)$ vanish. The expression of $d_{1}^{*}$ in (4.2) together with the formulas of Fact 3.1 tell us that $\Delta_{2}\left(U \mid u^{i+1}\right)$ is given by

$$
\frac{\left(r_{1}^{i+1}+r_{2}^{i+1}-\alpha\right)}{r_{1}} u^{i+1} w+r_{2}\left(r_{1}-r_{2}\right)\left(1-r_{2}^{i}\right) u^{i+2} d .
$$

Since the second coefficient of the previous expression is nonzero by the hypothesis of genericity, we see that the vanishing of $\Delta_{2}(x)$ implies that $a_{i+1}$ is zero, which we will assume from now on. We shall now turn our attention to $\Delta_{1}(x)$, for $x=$ $c_{i-1,1,0}^{\prime} D\left|u^{i-1} w+c_{i, 0,1}^{\prime} D\right| u^{i} d$. Using again the formulas of Fact 3.1 we see that

$$
\Delta_{1}\left(D \mid u^{i-1} w\right)=\frac{1-r_{2}^{i}}{r_{1}}\left(u^{i-1} w^{2}+r_{1}^{2}\left(r_{2}-r_{1}\right) u^{i} w d\right)
$$

and

$$
\Delta_{1}\left(D \mid u^{i} d\right)=\frac{\phi_{i-1}\left(r_{1}, r_{2}\right)}{r_{1}^{2}}\left(u^{i-1} w^{2}+r_{1}^{2}\left(r_{2}-r_{1}\right) u^{i} w d\right)
$$


The hypothesis of genericity implies that all the coefficients appearing in both of the previous expressions are nonzero. We note however that $\Delta_{1}\left(D \mid u^{i-1} w\right)$ and $\Delta_{1}\left(D \mid u^{i} d\right)$ are not linearly independent, and so $x$ is a multiple of $\phi_{i-1} D \mid u^{i-1} w-$ $r_{1}\left(1-r_{2}^{i}\right) D \mid u^{i} d$. Since $\phi_{i-1} D\left|u^{i-1} w-r_{1}\left(1-r_{2}^{i}\right) D\right| u^{i} d$ is also a multiple of $d_{0}^{*}\left(u^{i}\right)$ (see (4.8)), we conclude that the class of $x$ in $\left(V^{*} \otimes A\right) / \operatorname{Im}\left(d_{0}^{*}\right)$ vanishes. Case (ii) is analogous.

We now turn to a characterization of the space $H H^{2}(A(\alpha, \beta, 0))$. As before, let $A$ denote the algebra $A(\alpha, \beta, 0)$ and let $A(t, s)$ denote the Hilbert series of $A$ regarded as a bigraded algebra. This bigrading on $A$ induces a bigrading on its Hochschild cohomology, whose associated Hilbert series will be denoted by $H H^{i}(A)(t, s)$ for all $i \geq 0$.

Proposition 4.6. Under the previous assumptions,

$$
H H^{2}(A)(t, s)=\frac{1}{t^{2}}+2+\frac{t^{2}}{1-t^{2}} .
$$

Proof. Let $C^{\bullet}$ be the complex (4.1). Recall that the homology of $C^{\bullet}$ is $H H^{\bullet}(A)$. Regarding $H H^{\bullet}(A)$ as a complex with zero differentials, the Euler-Poincaré characteristic $\chi_{C} \bullet(t, s)$ associated to $C^{\bullet}$ is equal to the Euler-Poincaré characteristic $\left.\chi_{H H} \bullet{ }_{A}(t) s\right)$ associated to $H H^{\bullet}(A)$. Using the descriptions we obtained of $C^{\bullet}$ and of the Hochschild cohomology spaces $H H^{0}(A), H H^{1}(A)$ and $H H^{3}(A)$, the following equalities are straightforward to check,

$$
\begin{aligned}
& \chi_{C} \bullet(t, s)=-t^{-4}, \\
& H H^{0}(A)(t, s)=1, \\
& H H^{1}(A)(t, s)=2, \\
& H H^{3}(A)(t, s)=\frac{1}{t^{4}\left(1-t^{2}\right)} .
\end{aligned}
$$

Therefore, the equality $\chi_{C} \bullet(t, s)=\chi_{H_{\bullet}(A)}(t, s)$ is

$$
-\frac{1}{t^{4}}=1-2+H H^{2}(A)(t, s)-\frac{1}{t^{4}\left(1-t^{2}\right)},
$$

and the lemma follows.

From the previous lemma we deduce that every homogeneous component of $H H^{2}(A)$ of bidegree different from $(2 k, 0)$ for $k \geq-1$ is zero.

The following set is a $\mathbb{K}$-basis of the homogeneous component of $R^{*} \otimes A$ of bidegree $(2 k, 0)$

$$
\left\{D^{2} U \mid u^{a} w^{k+1-a} d^{a+1}: 0 \leq a \leq k+1\right\} \cup\left\{D U^{2} \mid u^{a+1} w^{k+1-a} d^{a}: 0 \leq a \leq k+1\right\} .
$$

Proposition 4.7. The homogeneous component of $H^{2}(A)$ of bidegree $(-2,0)$ is isomorphic to the $\mathbb{K}$-vector space spanned by the class of the element $D^{2} U\left|d+D U^{2}\right| u$. On the other hand, the homogeneous component of $H^{2}(A)$ of bidegree $(0,0)$ is isomorphic to the $\mathbb{K}$-vector space spanned by the classes of the elements $D^{2} U\left|w d+D U^{2}\right| u w$ and $D^{2} U\left|u d^{2}+D U^{2}\right| u^{2} d$.

Proof. There are no homogeneous elements of bidegree $(-2,0)$ in $V^{*} \otimes A$ and the homogeneous component of bidegree $(-2,0)$ in $R^{*} \otimes A$ is spanned by the elements $D^{2} U \mid d$ and $D U^{2} \mid u$. The first claim follows from the fact that $d_{2}^{*}\left(\lambda D^{2} U \mid d+\right.$ $\left.\mu D U^{2} \mid u\right)=(\mu-\lambda) D^{2} U^{2} \mid(d u+\beta u d)$.

The elements of $V^{*} \otimes A$ of bidegree $(0,0)$ are spanned by $D \mid d$ and $U \mid u$. We have already seen in Proposition 4.3 these elements are in the kernel of $d_{1}^{*}$. On the 
other hand, the elements $D^{2} U\left|w d+D U^{2}\right| u w$ and $D U^{2}\left|u d^{2}+D U^{2}\right| u^{2} d$ belong to the kernel of $d_{2}^{*}$ and they are linearly independent. By Proposition 4.6 their classes form a basis of the homogeneous component of $H H^{2}(A)$ of bidegree $(0,0)$.

We now turn to a description of the homogeneous components of bidegree $(2 k, 0)$ with $k \geq 1$. For all non negative integers $x, y$ and $z$, define

$$
\begin{aligned}
& g_{x, y, z}=d_{1}^{*}\left(U \mid u^{x} w_{1}^{y} d^{z}\right), \\
& f_{x, y, z}=d_{1}^{*}\left(D \mid u^{x} w_{1}^{y} d^{z}\right) .
\end{aligned}
$$

Observe that $\operatorname{bideg}\left(g_{x, y, z}\right)=(x+2 y+z-1, x-z-1)$ and $\operatorname{bideg}\left(f_{x, y, z}\right)=$ $(x+2 y+z-1, x-z+1)$.

Lemma 4.8. Let $k \geq 1$. The elements $f_{z, k-z, z+1}$, where $0 \leq z \leq k$, generate the homogeneous component of $\operatorname{Im}\left(d_{1}^{*}\right)$ of bidegree $(2 k, 0)$ as $\mathbb{K}$-vector space.

Proof. The set $\left\{U \mid u^{z+1} w_{1}^{k-z} d^{z}: 0 \leq z \leq k\right\} \cup\left\{D \mid u^{z} w_{1}^{k-z} d^{z+1}: 0 \leq z \leq k\right\}$ generates the homogeneous component of bidegree $(2 k, 0)$ of $V^{*} \otimes A$ and therefore the set $\left\{g_{z+1, k-z, z}: 0 \leq z \leq k\right\} \cup\left\{f_{z, k-z, z+1}: 0 \leq z \leq k\right\}$ generates the homogeneous component of $\operatorname{Im}\left(d_{1}^{*}\right)$ of the same bidegree.

For $0 \leq z \leq k$, define $x_{z}=U\left|u^{z+1} w_{1}^{k-z} d^{z}-D\right| u^{z} w_{1}^{k-z} d^{z+1}$. Observe that $d_{1}^{*}\left(x_{z}\right)=g_{z+1, k-z, z}-f_{z, k-z, z+1}$. Using the expression given in 4.8) for the image of an element under $d_{0}^{*}$ we see that

$$
d_{0}^{*}\left(u^{z} w_{1}^{k-z} d^{z}\right)=\left(1-r_{1}^{k-z} r_{2}^{z}\right) x_{z}-\frac{\phi_{z-1}}{r_{1}} x_{z-1} .
$$

Since $k \geq 1$, both coefficients appearing in the above equation are non zero. Taking $z=0$ and applying $d_{1}^{*}$, we deduce $d_{1}^{*}\left(x_{0}\right)=0$ and by an inductive argument we obtain $d_{1}^{*}\left(x_{z}\right)=0$ for all $0 \leq z \leq k$. As a consequence, $g_{z+1, k-z, z}=f_{z, k-z, z+1}$ for all $0 \leq z \leq k$ and the result follows.

Let $n$ and $m$ be the dimensions of the components of bidegree $(2 k, 0)$ of $\operatorname{Ker}\left(d_{2}^{*}\right)$ and $\operatorname{Im}\left(d_{1}^{*}\right)$, respectively. It is straightforward to check that

$$
d_{2}^{*}\left(D^{2} U\left|u^{a} w_{1}^{k+1-a} d^{a+1}+D U^{2}\right| u^{a+1} w_{1}^{k+1-a} d^{a}\right)=0,
$$

for all $0 \leq a \leq k+1$. We deduce that $n \geq k+2$. On the other hand, we know that $m \leq k+1$ by Lemma 4.8. Also, Proposition 4.6 implies $n-m=1$ and it follows that $n=k+2$ and $m=k+1$. As a consequence, the homogeneous component of $\operatorname{Ker}\left(d_{2}^{*}\right)$ of bidegree $(2 k, 0)$ is the $\mathbb{K}$-vector space spanned by the elements

$$
D^{2} U\left|u^{a} w_{1}^{k+1-a} d^{a+1}+D U^{2}\right| u^{a+1} w_{1}^{k+1-a} d^{a},
$$

for $0 \leq a \leq k+1$.

Let us prove that the element $D^{2} U\left|w_{1}^{k+1} d+D U^{2}\right| u w_{1}^{k+1}$ does not belong to the image $\operatorname{Im}\left(d_{1}^{*}\right)$. By definition, $f_{z, k-z, z+1}$ is equal to $d_{1}^{*}\left(D \mid u^{z} w_{1}^{k-z} d^{z+1}\right)$. We write $f_{z, k-z, z+1}=D^{2} U\left|\Delta_{1}\left(D \mid u^{z} w_{1}^{k-z} d^{z+1}\right)+D U^{2}\right| \Delta_{2}\left(D \mid u^{z} w_{1}^{k-z} d^{z+1}\right)$. For $0 \leq z \leq k$, Fact 3.1 implies that

$$
\begin{aligned}
\Delta_{2}\left(D \mid u^{z} w_{1}^{k-z} d^{z+1}\right)= & \frac{\phi_{z} \phi_{z-1}}{r_{1}^{2}} u^{z} w_{1}^{k-z+2} d^{z-1}+\frac{\left(r_{1}^{k-z} r_{2}^{z}-1\right) \alpha \phi_{z}}{r_{1}} u^{z+1} w_{1}^{k-z+1} d^{z} \\
& +\left(r_{1}^{k-z} r_{2}^{z+1}-r_{1}\right)\left(r_{1}^{k-z} r_{2}^{z+1}-r_{2}\right) u^{z+2} w_{1}^{k-z} d^{z+1} .
\end{aligned}
$$

Once more, the hypothesis of genericity implies that none of the coefficients appearing on the right hand side of the above equation annihilates. 
Suppose $D^{2} U\left|w_{1}^{k+1} d+D U^{2}\right| u w_{1}^{k+1}$ belongs to $\operatorname{Im}\left(d_{1}^{*}\right)$. By Lemma 4.8, there exist $\lambda_{0}, \ldots, \lambda_{k} \in \mathbb{K}$ such that

$$
D^{2} U\left|w_{1}^{k+1} d+D U^{2}\right| u w_{1}^{k+1}=\sum_{z=0}^{k} \lambda_{z} f_{z, k-z, z+1} .
$$

Therefore, $u w_{1}^{k+1}=\sum_{z=0}^{k} \lambda_{z} \Delta_{2}\left(D \mid u^{z} w_{1}^{k-z} d^{z+1}\right)$. Looking at the coefficient of $u^{k+2} d^{k+1}$ on the right hand side of the last equality, we deduce $\lambda_{k}=0$. Inductively in $z$ and looking at the coefficient of $u^{z+2} w_{1}^{k-z} d^{z+1}$, we deduce $\lambda_{z}=0$ for all $0 \leq z \leq k$, which is a contradiction. Thus, the element $D^{2} U\left|w_{1}^{k+1} d+D U^{2}\right| u w_{1}^{k+1}$ does not belong to $\operatorname{Im}\left(d_{1}^{*}\right)$. We have proven the following.

Proposition 4.9. Let $k \geq 1$. The homogeneous component of $\operatorname{Ker}\left(d_{2}^{*}\right)$ of bidegree $(2 k, 0)$ is the $\mathbb{K}$-vector space of dimension $k+2$ spanned by the set

$$
\left\{D^{2} U\left|u^{a} w_{1}^{k+1} d^{a+1}+D U^{2}\right| u^{a+1} w_{1}^{k+1} d^{a}: 0 \leq a \leq k+1\right\},
$$

and the class in $H H^{2}(A)$ of the element $D^{2} U\left|w_{1}^{k+1} d+D U^{2}\right| u w_{1}^{k+1}$ generates the homogeneous component of bidegree $(2 k, 0)$.

Theorem 2.2 follows from Propositions 4.14.2, 4.3, 4.6, 4.7 and 4.9

\section{References}

[1] Michael J. Bardzell, The alternating syzygy behavior of monomial algebras, J. Algebra 188 (1997), no. 1, 69-89. $\uparrow 2$

[2] Georgia Benkart, Down-up algebras and Witten's deformations of the universal enveloping algebra of $\mathfrak{s l}_{2}$, Recent progress in algebra (Taejon/Seoul, 1997), Contemp. Math., vol. 224, Amer. Math. Soc., Providence, RI, 1999 , pp. 29-45. $\uparrow 1$

[3] Georgia Benkart and Tom Roby, Down-up algebras, J. Algebra 209 (1998), no. 1, 305-344. $\uparrow 1,2$

[4] Georgia Benkart and Sarah Witherspoon, A Hopf structure for down-up algebras, Math. Z. 238 (2001), no. 3, 523-553. $\uparrow 1$

[5] Paula A. A. B. Carvalho and Samuel A. Lopes, Automorphisms of generalized down-up algebras, Comm. Algebra 37 (2009), no. 5, 1622-1646. $\uparrow 1$

[6] Paula A. A. B. Carvalho and Ian M. Musson, Down-up algebras and their representation theory, J. Algebra 228 (2000), no. 1, 286-310. $\uparrow 1,2$

[7] Thomas Cassidy and Brad Shelton, Basic properties of generalized down-up algebras, J. Algebra 279 (2004), no. 1, 402-421. $\uparrow 1,2$

[8] Sergio Chouhy and Andrea Solotar, Projective resolutions of associative algebras and ambiguities, J. Algebra 432 (2015), 22-61. $\uparrow 1,3$

[9] S. V. Fomin, The generalized Robinson-Schensted-Knuth correspondence, Zap. Nauchn. Sem. Leningrad. Otdel. Mat. Inst. Steklov. (LOMI) 155 (1986), no. Differentsialnaya Geometriya, Gruppy Li i Mekh. VIII, 156-175, 195 (Russian); English transl., J. Soviet Math. 41 (1988), no. 2, 979-991. $\uparrow 1$

[10] Thomas Goodwillie, Cyclic homology, derivations and the free loop spaceBasic properties of generalized down-up algebras, Topology 24 (2004), no. 2, 187-215. $\uparrow 7$

[11] Estanislao Herscovich and Andrea Solotar, Hochschild and cyclic homology of Yang-Mills algebras, J. Reine Angew. Math. 665 (2012), 73-156. 11,2

[12] K. Igusa, Cyclic homology and the determinant of the Cartan matrix, J. Pure Appl. Algebra 83 (1992), 101-119. $\uparrow 3,7$

[13] Ellen Kirkman, Ian M. Musson, and D. S. Passman, Noetherian down-up algebras, Proc. Amer. Math. Soc. 127 (1999), no. 11, 3161-3167. $\uparrow 2$

[14] Rajesh S. Kulkarni, Down-up algebras and their representations, J. Algebra 245 (2001), no. 2, 431-462. $\uparrow 1,2,3,12$

[15] Yuan Shen and DiMing Lu, Nakayama automorphisms of PBW deformations and Hopf actions, Sci. China Math. 59 (2016), no. 4, 661-672. $\uparrow 4$

[16] Richard P. Stanley, Differential posets, J. Amer. Math. Soc. 1 (1988), no. 4, 919-961. $\uparrow 1$ 
[17] Xin Tang, Algebra endomorphisms and derivations of some localized down-up algebras, J. Algebra Appl. 14 (2015), no. 3, 1550034, 14. $\uparrow 2$

[18] Paul Terwilliger, The incidence algebra of a uniform poset, Coding theory and design theory, Part I, IMA Vol. Math. Appl., vol. 20, Springer, New York, 1990, pp. 193-212. $\uparrow 1$

[19] Micheline Vigué-Poirrier, Cyclic homology of algebraic hypersurfaces, J. Pure Appl. Algebra 72 (1991), 95-108. $\uparrow 7$

[20] Kaiming Zhao, Centers of down-up algebras, J. Algebra 214 (1999), 103-121. ^1, 2, 3, 6

S.C.:

IMAS, UBA-CONICET, Consejo Nacional de Investigaciones Cientícas y Técnicas,

Ciudad Universitaria, Pabellón I, 1428 Buenos Aires, Argentina

schouhyedm.uba.ar

E.H.:

Institut Fourier, Université Grenoble Alpes,

100 rue des Maths, 38610 Gières, France;

Departamento de Matemática, Facultad de Ciencias Exactas y Naturales, Universidad de Buenos Aires, Ciudad Universitaria, Pabellón I, 1428 Buenos Aires, Argentina; and

IMAS, UBA-CONICET, Consejo Nacional de Investigaciones Científicas y Técnicas, Argentina

Estanislao.Herscovich@univ-grenoble-alpes.fr

A.S.:

Departamento de Matemática, Facultad de Ciencias Exactas y Naturales, Universidad de Buenos Aires, Ciudad Universitaria, Pabellón I, 1428 Buenos Aires, Argentina; and

IMAS, UBA-CONICET, Consejo Nacional de Investigaciones Científicas y Técnicas, Argentina

asolotaredm.uba.ar 